\title{
BMJ Open Effects of moxibustion on symptoms of mild cognitive impairment: protocol of a systematic review and meta-analysis
}

\author{
Tao Zhang (D) , ${ }^{1}$ Lin-Peng Wang, ${ }^{1}$ Gui-Ling Wang, ${ }^{1}$ Jing-Qing Sun, ${ }^{1}$ Xue-Wen Mao, ${ }^{2}$ \\ Hui-Li Jiang, ${ }^{3}$ Bin Li, ${ }^{1}$ Cun-Zhi Liu ${ }^{3}$
}

To cite: Zhang T, Wang L-P, Wang G-L, et al. Effects of moxibustion on symptoms of mild cognitive impairment: protocol of a systematic review and meta-analysis. BMJ Open 2020;10:e033910. doi:10.1136/ bmjopen-2019-033910

- Prepublication history for this paper is available online. To view these files, please visit the journal online (http://dx.doi. org/10.1136/bmjopen-2019033910).

$\mathrm{BL}$ and C-ZL contributed equally.

Received 29 August 2019 Revised 28 January 2020 Accepted 24 March 2020

\section{Check for updates}

(c) Author(s) (or their employer(s)) 2020. Re-use permitted under CC BY-NC. No commercial re-use. See rights and permissions. Published by BMJ.

${ }^{1}$ Department of Acupuncture and Moxibustion, Beijing Hospital of Traditional Chinese Medicine, Capital Medical University, Beijing Key Laboratory of Acupuncture Neuromodulation, Beijing, China

${ }^{2}$ Department of Acupuncture and Moxibustion, Shunyi Hospital, Beijing Traditional Chinese

Medicine Hospital, Beijing, China

${ }^{3}$ Acupuncture Research Center, School of AcupunctureMoxibustion and Tuina, Beijing University of Chinese Medicine, Beijing, China

Correspondence to Dr Cun-Zhi Liu; Icz623780@126.com and Dr Bin Li;

libin@bjzhongyi.com

\section{ABSTRACT}

Introduction Mild cognitive impairment (MCl) is considered the intermediate phase between normal agerelated cognitive decline and dementia. Moxibustion has gained increased popularity for the management of $\mathrm{MCl}$ in China.This study aimed to evaluate the effects and safety of moxibustion on symptoms of $\mathrm{MCl}$.

Methods and analysis Four English databases and six Chinese databases will be searched from their inception to October 2019: Embase, MEDLINE, Cochrane Central Register of Controlled Trials, Allied and Complementary Medicine Database, China National Knowledge Infrastructure, Chongqing VIP Chinese Science and Technology Periodical Database, Wanfang Database, SinoMed, China Doctoral Dissertations Full-text Database and the China Master's Theses Full-text Database. Only clinical randomised controlled trials and the first period in randomised cross-over trial related to moxibustion for $\mathrm{MCl}$ will be included. The primary outcomes include the improvement of cognitive function, as measured by validated assessment tools. The secondary outcomes include changes in the activity of daily living scale, effective rate and the incidences of adverse events. The selection of studies, data extraction and risk of bias assessment will be carried out by two independent reviewers. Review Manager V.5.3 software will be used for statistical analyses. Heterogeneity test, data synthesis and subgroup analysis will be performed if necessary. The risk of bias of included studies will be assessed by the Cochrane Handbook risk of bias tool. Evidence quality will be evaluated using the Grading of Recommendations Assessment, Development and Evaluation system.

Ethics and dissemination Ethics approval is not required as no private information from individuals are collected. The results will be published in a peer-reviewed journal or disseminated in relevant conferences.

Trial registration number CRD42018112657.

\section{INTRODUCTION}

Mild cognitive impairment (MCI) is characterised by deterioration of cognitive function, ${ }^{1}$ with minimal impairment of activities of daily living (ADLs). ${ }^{2}$ It is also considered the intermediate phase between normal agerelated cognitive decline and dementia. ${ }^{3}$ MCI affects about 21.5-71.3 per 1000 adults and is reported to have an incidence of

\section{Strengths and limitations of this study}

This study will be the first systematic review and meta-analysis that investigated the effects of moxibustion on symptoms of mild cognitive impairment.

- This study will employ strict inclusion and exclusion criteria to search for randomised controlled trial in four English databases and six Chinese databases.

- Any missing or inadequate data will be obtained by contacting the first or corresponding author of included studies.

Different types of moxibustion therapy may lead to a large degree of heterogeneity and make data synthesis more difficult.

$6 \%-42 \%$ in the elderly worldwide each year. ${ }^{56}$ Its prevalence increases with age and is associated with lower educational levels. ${ }^{6}$ The severity of MCI involvement varies and the risk of MCI conversion to dementia is $10 \%-15 \%$ per year, ${ }^{7-9}$ further increasing the societal burden of this condition.

It is estimated that the prevalence of MCI is significantly increasing worldwide, and MCI increases the risk of progression to dementia. ${ }^{9}$ Accordingly, conceptual frameworks for new preventive strategies and effective therapies for treating MCI are urgently needed. Currently, strategies to prevent MCI include stress reduction, avoidance of toxins, and treatment for neurological, neurodegenerative and systemic disorders. ${ }^{11} 11$ Non-pharmacological interventions, such as physical activity programmes, cognitive rehabilitation, psychological treatments, aromatherapy and light therapy have been attempted as well. ${ }^{12}$ However, evidence as to the efficacy and safety of these therapies is limited.

Moxibustion is a form of traditional Chinese medicine that has been widely used in East Asia for thousands of years. ${ }^{13}$ It involves the burning of dried mugwort leaves at one or more relevant acupoints, or on the handle of an acupuncture needle after insertion. ${ }^{14}$ 
Moxibustion is painless, easy to operate and associated with few adverse events. ${ }^{15}$ Additionally, patients can perform it at home. Moxibustion imparts both heat stimulation via infrared radiation and the pharmacological actions of its herbal components. ${ }^{16}{ }^{17}$ It regulates a multidimensional network that includes the nervous, endocrine and immune systems, all of which play important roles in maintaining homeostasis, potentially exerting significant therapeutic effects. ${ }^{18-22}$ Various clinical trials and animal studies, mostly out of China, have been conducted to investigate the benefits and mechanisms of moxibustion for preventing and treating MCI. ${ }^{23} 24$

In spite of its long history of use and clinical and experimental support, the effects of moxibustion for MCI have not been fully validated. Additionally, systematic reviews and meta-analyses have not been done. Thus, this systematic review and meta-analysis aimed to assess the effects and safety of moxibustion on symptoms of MCI.

\section{METHODS}

\section{Criteria for including studies in the review}

Types of studies

The review will include randomised controlled trials (RCTs) and the first period in randomised cross-over trials, regardless of publication status. Studies without comparable baselines and duplicate publications will be removed.

\section{Types of participants}

Participants with all types of MCI, including amnestic mild cognitive impairment (aMCI), non-aMCI and vascular MCI, who meeting the diagnostic criteria of MCI will be included. These criteria include the Diagnostic and Statistical Manual of Mental Disorders, International Classification of Diseases, diagnostic criteria from the International Working Group on Mild Cognitive Impairment, diagnostic criteria from the MCI Working Group of the European Consortium on Alzheimer's Disease and the Chinese Classification of Mental Disorders. There is no restriction on age, gender, ethnicity or economic status of the enrolled participants.

\section{Types of interventions}

Interventions in the treatment group will include any kind of moxibustion treatment, regardless of manipulation. These include moxa cone moxibustion, moxa stick moxibustion, moxa burner moxibustion, medicine thread moxibustion and warm needling. We also include moxibustion in combination with other conservative treatments, such as acupuncture therapy, cognitive rehabilitation and oral drugs. However, combined interventions consisting of three or more therapies or with potential safety problems will be excluded.

Control interventions will include no treatment, waiting list, oral drugs, cognitive rehabilitation and sham moxibustion.
Types of outcomes

The primary outcome of the study is the improvement of cognitive function, as measured by validated assessment tools. The assessment tools include the Mini-Mental State of Examination, Montreal Cognitive Assessment, Global Deterioration Scale, Clinical Memory Scale and Hasegawa Dementia Scale.

The secondary outcomes included changes in the ADL, clinical efficacy and the incidences of adverse events related to moxibustion (such as allergy, scalding, local infection, coughing and nausea).

\section{Search methods for identification of studies Electronic searches}

The following databases will be searched from their inception until October 2019: Embase, MEDLINE, Cochrane Central Register of Controlled Trials, Allied and Complementary Medicine Database, China National Knowledge Infrastructure, Chongqing VIP Chinese Science and Technology Periodical Database, Wanfang Database, SinoMed, China Doctoral Dissertations Full-text Database and the China Master's Theses Full-text Database. Only RCTs that investigate the effects of moxibustion for $\mathrm{MCI}$ in comparison with the aforementioned comparator controls will be included. The following Medical Search Headings (MeSH) will be used: mild cognitive impairment, cognitive impairment, cognitive dysfunctions, mild neurocognitive disorder, cognitive decline, moxibustion, mugwort, artemisia, moxa, randomised controlled trial, randomised controlled, randomised, controlled, clinical trial, comparative study and prospective study. Chinese translations of these search terms will be used for the Chinese databases. The search strategy for MEDLINE is listed in table 1 . The search strategy for other online databases will be adjusted according to their requirements.

\section{Searching other resources}

The reference lists of all included studies or relevant reports of clinical trials or reviews will be screened for additional relevant articles. The WHO International Clinical Trials Registry Platform, Chinese Clinical Trial Registry and ClinicalTrials. gov will also be searched to identify the ongoing or unpublished trials.

\section{Data collection and analysis}

Selection of studies

Clinical studies will be identified and reviewed by two independent reviewers (G-LW and J-QS). The reviewers will read the titles, abstracts and, if necessary, full texts, and collect the studies that meet inclusion criteria. Applicable RCTs will be selected and cross-checked by the two reviewers (G-LW and J-QS). Disagreements related to study selection will be discussed after cross-checking and adjudicated by a third reviewer (L-PW). The process of study selection is presented in a Preferred Reporting Items for Systematic Reviews and Meta-Analyses flow diagram (figure 1). 


\begin{tabular}{|c|c|}
\hline Number & Search terms \\
\hline$\# 1$ & randomised controlled trial.pt. \\
\hline \#2 & controlled clinical trial.pt. \\
\hline \#3 & randomised.ab. \\
\hline \#4 & randomised.ab. \\
\hline \#5 & placebo.ab. \\
\hline \#6 & randomly.ab. \\
\hline \#7 & trial.ab. \\
\hline \#8 & clinical trials as topic (MeSH) \\
\hline$\# 9$ & \#1 OR \#2 OR \#3 OR \#4 OR \#5 OR \#6 OR \#7 OR \#8 \\
\hline \#10 & cognitive impairment (MeSH) \\
\hline \#11 & neurocognitive disorder (MeSH) \\
\hline \#12 & cognitive dysfunction (MeSH) \\
\hline \#13 & cognitive decline $(\mathrm{MeSH})$ \\
\hline \#14 & \#10 OR \#11 OR \#12 OR \#13 \\
\hline \#15 & moxibustion (MeSH) \\
\hline \#16 & artemisia $(\mathrm{MeSH})$ \\
\hline \#17 & moxa.ab. \\
\hline \#18 & mugwort.ab. \\
\hline \#19 & \#15 OR \#16 OR \#17 OR \#18 \\
\hline \#20 & \#9 AND \#14 AND\#19 \\
\hline \#21 & remove duplicates from \#20 \\
\hline
\end{tabular}

\section{Data extraction and management}

A predefined data extraction form will be developed based on Cochrane Handbook V.5.1.0 and independently completed by each of the two reviewers (X-WM and H-LJ).

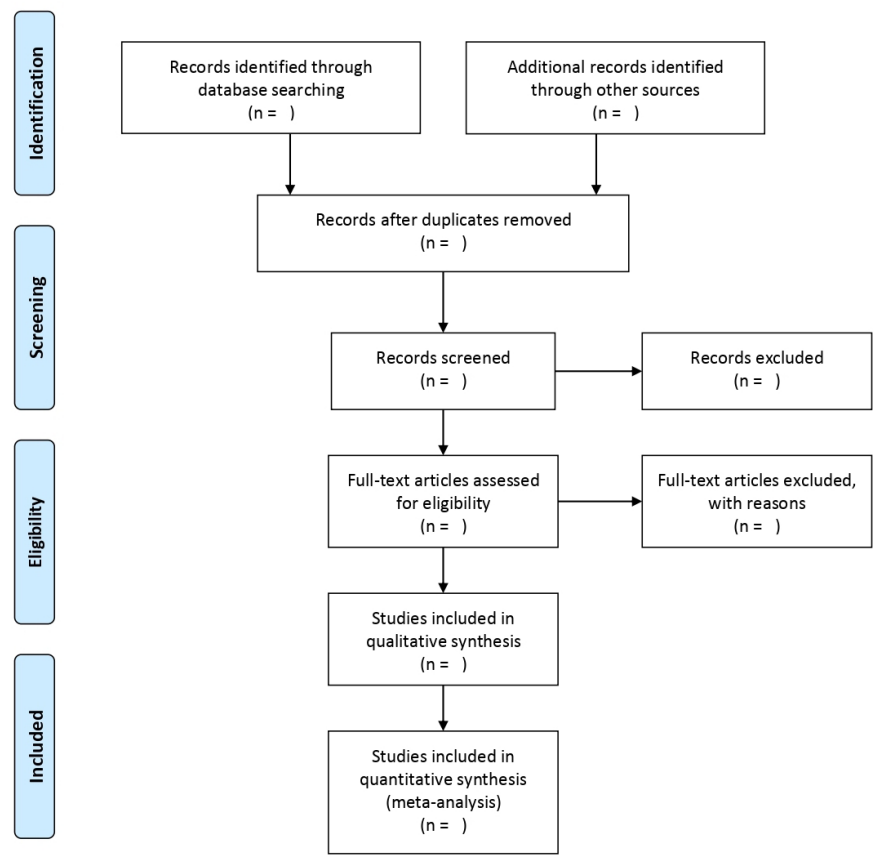

Figure 1 PRISMA flow diagram of the study process. PRISMA, Preferred Reporting Items for Systematic Reviews and Meta-Analyses.
The data extraction form contains information about the participants, interventions, outcomes, adverse events, randomisation, allocation concealment, incomplete data, blinding, selective report, conflict of interest and selfanalysis of risk of bias by the author. The data extraction results will be cross-checked, and any disagreements will be discussed and adjudicated by a third reviewer (C-ZL).

\section{Assessment of risk of bias in included studies}

According to the methods for assessing risk of bias using the Cochrane Handbook 'risk of bias' tool V.5.1.0, the following types of bias will be assessed: selection bias (random sequence generation and allocation concealment), performance bias (blinding of participants, investigators and care providers), detection bias (blinding of outcome assessment), attrition bias (incomplete data or differential dropout), reporting bias (selective reporting) and other biases (conflict of interest). ${ }^{25} 26$ These will be independently evaluated by two reviewers (TZ and BL). The result of the assessment will be cross-checked. Any disagreement will be discussed and adjudicated by a third reviewer (L-PW).

\section{Measures of treatment effect}

Review Manager statistical software (RevMan) V.5.3 will be used for data synthesis and analysis. Dichotomous data will be analysed by using a risk ratio with $95 \%$ CIs. For continuous outcomes, data will be analysed by using a mean difference with $95 \%$ CIs.

\section{Unit of analysis issues}

The units of each outcome from different studies will be converted to the International System of Units before statistical analysis.

\section{Dealing with missing data}

If required data are inadequate or not reported in the studies, reviewers will contact the first or corresponding author of those studies by phone or email. If missing data are unobtainable, those studies will be excluded.

\section{Assessment of heterogeneity}

Prior to the meta-analysis, we will assess study heterogeneity using the Mantel-Haenszel $\chi^{2}$ test and I square $\left(\mathrm{I}^{2}\right)$ statistic of homogeneity by RevMan. A low $\mathrm{p}$ value $(\mathrm{p}<0.10)$ and a high $\mathrm{I}^{2}$ value indicated statistically significant heterogeneity. According to the Cochrane Handbook, $\mathrm{I}^{2}$ value can be classified into four categories: a value of $0 \%-40 \%$ indicates little ornoheterogeneity; a value of $30 \%-60 \%$ indicates moderate heterogeneity; a value of $50 \%-90 \%$ indicates substantial heterogeneity; and a value of $75 \%-100 \%$ indicates considerable heterogeneity. ${ }^{25}$

\section{Assessment of reporting biases}

A funnel plot will be generated to observe the reporting bias when more than 10 studies are included. The plots will be assessed visually and by using Egger's test. ${ }^{27}$ 
Data synthesis

RevMan software will be used for data synthesis. Two or more eligible studies with low heterogeneity $(p \geq 0.10$ and $\mathrm{I}^{2}<50 \%$ ) will be combined for the purpose of metaanalysis, and the results will be pooled using a fixed-effects model. If the $\mathrm{p}$ value is $<0.10$ or $\mathrm{I}^{2}$ is $\geq 50 \%$, the results will be combined using a random-effects model. If the metaanalysis is not feasible due to considerable clinical and methodological heterogeneity, a qualitative summary of the results will be conducted. ${ }^{28}$

\section{Subgroup analysis and investigation of heterogeneity}

Subgroup analysis will be performed to detect possible causes of heterogeneity in the previous analysis if necessary. It will be conducted according to variations in the characteristics of the participants, moxibustion treatments and controls.

\section{Sensitivity analysis}

Sensitivity analysis will be conducted to monitor the robustness of pooled treatment effects as suggested by the Cochrane Handbook. The meta-analysis will be repeated by excluding studies with small sample size, methodological weaknesses and missing data. If inconsistent results are identified, it will be discussed and caution will be taken in drawing conclusions.

\section{Evidence quality evaluation}

The Grading of Recommendations Assessment, Development and Evaluation (GRADE) profiler, provided by the GRADE Working Group (www.gradeworkinggroup.org), will be used by two reviewers (G-LW and J-QS) to independently assess the methodological quality of included studies. Study limitations, inconsistency of results, indirectness of evidence, imprecision and publication bias will be evaluated to determine if their effects on the quality of evidence are negligible, serious or very serious, in accordance with the GRADE and Cochrane Handbook V.5.1.0 criteria. ${ }^{29}{ }^{30}$ Evidence quality will be rated into one of four possible ratings: high, moderate, low and very low. The assessment results will also be cross-checked, and any disagreements will be discussed with, and adjudicated by, a third reviewer (BL).

\section{Patient and public involvement}

There was no patient or public involvement in the preparation of the study protocol. Patients will not be involved in the data collection and analysis of the systematic review and meta-analysis.

\section{Amendments}

The information of any amendment will be described in the final report.

\section{Ethics and dissemination}

Ethics approval is not required as the study will not collect private information from individuals or violate their personal rights. The results of this study will provide a general overview and evidence of the effects and safety of moxibustion on symptoms of MCI. The findings will be published in a peer-reviewed journal or disseminated in relevant conferences.

Acknowledgements We acknowledge Professor Marc Fisher, a distinguished professor of Beijing Municipal Government from Harvard Medical School, who helped us revise the manuscript.

Contributors TZ, BL and C-ZL conceived the study. TZ and C-ZL drafted the manuscript. BL and L-PW designed the search strategy. G-LW, J-QS, X-WM and $H$-LJ reviewed and revised the manuscript. All authors have read and approved the final version of the manuscript.

Funding This work was supported by the following organisations and funds: Fostering Talents of Beijing Municipal Government, Beijing, China (code 2017000021469G298); Fostering Talents of Beijing Hospital of Traditional Chinese Medicine, Capital Medical University, Beijing, China (code XX-201702); Beijing Hospitals Authority Youth Programme, Beijing, China (code QML20191001); Beijing Municipal Administration of Hospitals Incubating Program, Beijing, China (code PZ2020025); China Clinical Talents Project of Traditional Chinese Medicine (the Fourth Batch), National Administration of Traditional Chinese Medicine, Beijing, China (code [2017]24).

Competing interests None declared.

Patient and public involvement Patients and/or the public were not involved in the design, or conduct, or reporting, or dissemination plans of this research.

Patient consent for publication Not required.

Provenance and peer review Not commissioned; externally peer reviewed.

Open access This is an open access article distributed in accordance with the Creative Commons Attribution Non Commercial (CC BY-NC 4.0) license, which permits others to distribute, remix, adapt, build upon this work non-commercially, and license their derivative works on different terms, provided the original work is properly cited, appropriate credit is given, any changes made indicated, and the use is non-commercial. See: http://creativecommons.org/licenses/by-nc/4.0/.

ORCID iD

Tao Zhang http://orcid.org/0000-0002-8349-9091

\section{REFERENCES}

1 Petersen RC, Doody R, Kurz A, et al. Current concepts in mild cognitive impairment. Arch Neurol 2001;58:1985-92.

2 Petersen RC. Mild cognitive impairment: current research and clinical implications. Semin Neurol 2007;27:022-31.

3 Albert MS, DeKosky ST, Dickson D, et al. The diagnosis of mild cognitive impairment due to Alzheimer's disease: recommendations from the National Institute on Aging-Alzheimer's association workgroups on diagnostic guidelines for Alzheimer's disease. Alzheimers Dement 2011;7:270-9.

4 Odawara T. Cautious notification and continual monitoring of patients with mild cognitive impairment. Psychogeriatrics 2012;12:131-2.

5 Tricco AC, Soobiah C, Lillie E, et al. Use of cognitive enhancers for mild cognitive impairment: protocol for a systematic review and network meta-analysis. Syst Rev 2012;1:25.

6 Petersen RC, Lopez O, Armstrong MJ, et al. Practice guideline update summary: mild cognitive impairment: report of the Guideline development, Dissemination, and Implementation Subcommittee of the American Academy of Neurology. Neurology 2018;90:126-35.

7 DeCarli C. Mild cognitive impairment: prevalence, prognosis, aetiology, and treatment. Lancet Neurol 2003;2:15-21.

8 Ritchie K, Touchon J. Mild cognitive impairment: conceptual basis and current nosological status. Lancet 2000;355:225-8.

9 Mitchell AJ, Beaumont H, Ferguson D, et al. Risk of dementia and mild cognitive impairment in older people with subjective memory complaints: meta-analysis. Acta Psychiatr Scand 2014;130:439-51.

10 Feldman $\mathrm{HH}$, Ferris S, Winblad B, et al. Effect of rivastigmine on delay to diagnosis of Alzheimer's disease from mild cognitive impairment: the InDDEx study. Lancet Neurol 2007;6:501-12.

11 Birks J, Flicker L. Donepezil for mild cognitive impairment. Cochrane Database Syst Rev 2006;19:CD006104.

12 Vilela VC, Pacheco RL, Latorraca COC, et al. What do Cochrane systematic reviews say about non-pharmacological interventions for treating cognitive decline and dementia? Sao Paulo Med J 2017;135:309-20. 
13 Shen X, Ding G, Wei J, et al. An infrared radiation study of the biophysical characteristics of traditional moxibustion. Complement Ther Med 2006;14:213-9.

14 Pach D, Brinkhaus B, Willich SN. Moxa sticks: thermal properties and possible implications for clinical trials. Complement Ther Med 2009;17:243-6.

$15 \mathrm{Xu}$ J, Deng H, Shen X. Safety of moxibustion: a systematic review of case reports. Evid Based Complement Alternat Med 2014;2014:1-10

16 Okada K, Kawakita K. Analgesic action of acupuncture and moxibustion: a review of unique approaches in Japan. Evid Based Complement Alternat Med 2009;6:11-17.

17 Kawakita K, Shinbara H, Imai K, et al. How do acupuncture and moxibustion act? - Focusing on the progress in Japanese acupuncture research -. J Pharmacol Sci 2006;100:443-59.

18 Bian X-M, Lv L, Lin W-B, et al. Moxibustion therapy at CV4 prevents postoperative dysuria after procedure for prolapse and hemorrhoids. Evid Based Complement Alternat Med 2013;2013:1-5.

19 Yang M, Chen X, Bo L, et al. Moxibustion for pain relief in patients with primary dysmenorrhea: a randomized controlled trial. PLoS One 2017;12:e0170952.

20 Huang Z, Qin Z, Yao Q, et al. Moxibustion for chemotherapy-induced nausea and vomiting: a systematic review and meta-analysis. Evid Based Complement Alternat Med 2017;2017:1-11.

21 Xu F, Huang M, Jin Y, et al. Moxibustion treatment for primary osteoporosis: a systematic review of randomized controlled trials. PLoS One 2017;12:e0178688.

22 Tang B, Zhang J, Yang Z, et al. Moxibustion for diarrhea-predominant irritable bowel syndrome: a systematic review and meta-analysis of randomized controlled trials. Evid Based Complement Alternat Med 2016;2016:1-10

23 Yang $\mathrm{X}$, Wang $\mathrm{Y}$, Zhang $\mathrm{H}$. Research situation on moxibustion in the treatment of mild cognitive impairment and dementia. Guangming journal of Chinese Medicine 2016;31:1506-8.

24 Choe S, Cai M, Jerng UM, et al. The efficacy and underlying mechanism of moxibustion in preventing cognitive impairment: a systematic review of animal studies. Exp Neurobiol 2018;27:1-15.

25 The Cochrane CollaborationHiggins JPT, Green S, eds. Cochrane Handbook for systematic reviews of interventions. Version 5.1.0. The Cochrane Collaboration, 2011.

26 Zeng X, Zhang Y, Kwong JSW, et al. The methodological quality assessment tools for preclinical and clinical studies, systematic review and meta-analysis, and clinical practice guideline: a systematic review. J Evid Based Med 2015;8:2-10.

27 Aamann L, Dam G, Rinnov A, et al. Physical exercise for people with cirrhosis. Cochrane Database Syst Rev 2017;6:CD012678.

28 Schilder AG, Chong LY, Ftouh S, et al. Bilateral versus unilateral hearing AIDS for bilateral hearing impairment in adults. Cochrane Database Syst Rev 2017;12:CD012665

29 Guyatt GH, Oxman AD, Vist GE, et al. Grade: an emerging consensus on rating quality of evidence and strength of recommendations. BMJ 2008;336:924-6.

30 Guyatt GH, Oxman AD, Schünemann HJ, et al. Grade guidelines: a new series of articles in the Journal of clinical epidemiology. J Clin Epidemiol 2011;64:380-2. 